\title{
Aero-Acoustics of Drag Generating Swirling Exhaust Flows
}

\author{
P. N. Shah ${ }^{*}$, D. Mobed ${ }^{\dagger}$, Z. S. Spakovszky ${ }^{\ddagger}$ \\ Gas Turbine Laboratory, Massachusetts Institute of Technology, Cambridge, MA, 02139 \\ T. F. Brooks ${ }^{\S}$, W. M. Humphreys, Jr. ${ }^{* *}$ \\ NASA Langley Research Center, Hampton, VA, 23681
}

\begin{abstract}
Aircraft on approach in high-drag and high-lift configuration create unsteady flow structures which inherently generate noise. For devices such as flaps, spoilers and the undercarriage there is a strong correlation between overall noise and drag such that, in the quest for quieter aircraft, one challenge is to generate drag at low noise levels. This paper presents a rigorous aero-acoustic assessment of a novel drag concept. The idea is that a swirling exhaust flow can yield a steady, and thus relatively quiet, streamwise vortex which is supported by a radial pressure gradient responsible for pressure drag. Flows with swirl are naturally limited by instabilities such as vortex breakdown. The paper presents a first aero-acoustic assessment of ram pressure driven swirling exhaust flows and their associated instabilities. The technical approach combines an in-depth aerodynamic analysis, plausibility arguments to qualitatively describe the nature of acoustic sources, and detailed, quantitative acoustic measurements using a medium aperture directional microphone array in combination with a previously established Deconvolution Approach for Mapping of Acoustic Sources (DAMAS). A model scale engine nacelle with stationary swirl vanes was designed and tested in the NASA Langley Quiet Flow Facility at a full-scale approach Mach number of 0.17. The analysis shows that the acoustic signature is comprised of quadrupole-type turbulent mixing noise of the swirling core flow and scattering noise from vane boundary layers and turbulent eddies of the burst vortex structure near sharp edges. The exposed edges are the nacelle and pylon trailing edge and the centerbody supporting the vanes. For the highest stable swirl angle setting a nacelle area based drag coefficient of 0.8 was achieved with a full-scale OASPL of about 40dBA at the ICAO approach certification point.
\end{abstract}

\section{Background \& Introduction}

Q WIRLING flows are involved in many fluid $\checkmark$ machinery applications ranging from combustion chambers, turbomachines and their associated ducting, and cyclone separators. A novel application is based on the concept of exploiting the low pressure in the vortex cores to generate drag. The idea is that a swirling exhaust flow, for example emanating from an engine nacelle as shown in Figure 1, can yield a steady streamwise vortex in which the centripetal acceleration of fluid particles is balanced by a radial pressure gradient. The very low pressure near the vortex core at the exit of the duct generates pressure drag. The

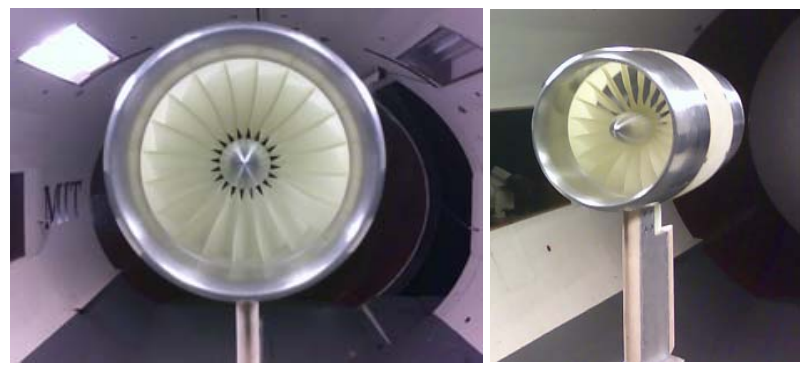

Figure 1. Aerodynamic flow testing and drag measurements of swirling exhaust flows ${ }^{1,2}$. aerodynamic analysis of this concept is described in detail in Shah ${ }^{1}$ and Shah et al. ${ }^{2}$. The simplest possible

\footnotetext{
${ }^{*}$ Ph.D. Student, Department of Aeronautics and Astronautics, 77 Massachusetts Ave, Member AIAA.

${ }^{\dagger}$ M.S. Student, Department of Aeronautics and Astronautics, 77 Massachusetts Ave, Member AIAA.

${ }^{\ddagger}$ Associate Professor, Department of Aeronautics and Astronautics, 77 Massachusetts Ave, Member AIAA.

$\S$ Senior Research Scientist, Aeroacoutics Branch, Fellow AIAA

** Senior Research Scientist, Aeroacoustics Branch, , Senior Member AIAA.
} 
configuration is a ram-pressure driven nacelle with a row of stationary swirl vanes (no rotating parts) as depicted in Figure 1. The stability limit of such a swirling flow is set by vortex breakdown phenomena which are predicted to occur at swirl angles in excess of 50 degrees (for an in-depth discussion see Shah $^{1}$ and Greitzer et al. ${ }^{3}$ ). Aerodynamic wind tunnel tests of this device were conducted using hot-wire anemometry and flow visualization validating the predicted instability threshold. The device was mounted on a drag balance and a maximum drag coefficient of 0.83 based on nacelle area was measured as shown by the blue curve in Figure 2. Although high drag capability is demonstrated the major unknowns are the acoustic signature of such swirling flow fields and the impact of vortex breakdown on the noise levels. It is important to note that the flow investigated here is markedly different from a swirling jet. The ram-air driven nacelle with swirl vanes sets up a wake-like flow with a streamwise vortex. The acoustic assessment of such drag generating swirling exhaust flows has not yet been reported and is the focus of this paper.

Related previous work is that of acoustic sources in circular motion and noise from swirling jets. Tanna and Morfey ${ }^{4}$ evaluated the sound radiated in the far field due to point sources of random time variation rotating uniformly in a circle at subsonic speed. This approach was extended by

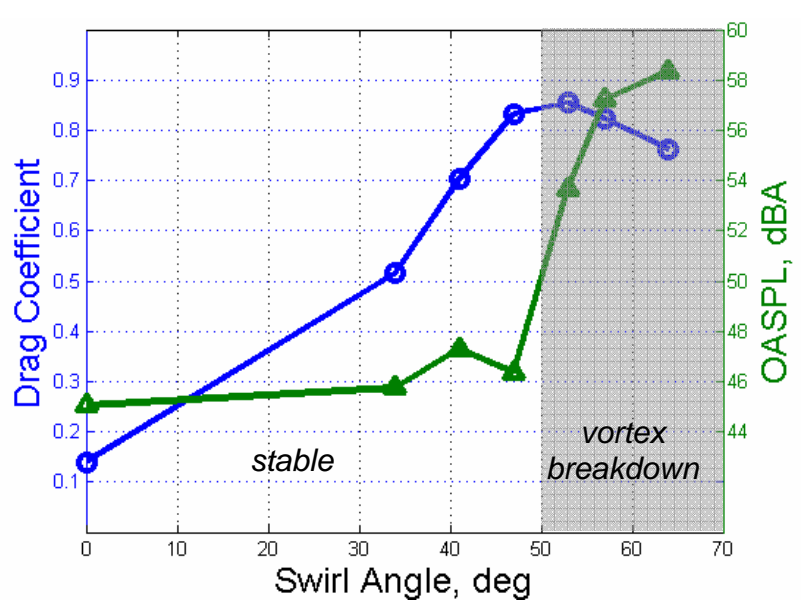

Figure 2. Experimentally measured drag coefficient (blue) and OASP (green) for swirling exhaust flows ${ }^{1,2}$.

$\operatorname{Tanna}^{5}$ to assess the effect of swirling motion of sources on subsonic jet noise. It was found that the effect is maximum at 90 degrees to the jet axis whereas the on-axis radiation form a randomly oriented point quadrupole moving uniformly on a helical path is independent of swirl. The magnitude of the effect of swirl increases with swirl angle. Jet noise suppression by swirling the jet flow was stimulated by Schwartz ${ }^{6}$ who obtained a ratio of 3dB overall sound power reduction to $1 \%$ of thrust loss for a Pratt \& Whitney JT15D-1 bypass flow engine by swirling a part of the primary flow. Lu et al. ${ }^{7}$ measured the noise and flow characteristics of model swirling jets and report that swirling jet noise is broadband in nature similar to non-swirling jet noise. The noise levels increase with swirl angle and decrease with increasing pressure ratio and total temperature. They conclude that considerable further testing of swirling jet flow and noise, especially under the influence of a parallel mean flow, is required to advance the understanding of the acoustic signature of such exhaust flows.

The objectives of this paper are to (1) quantify the noise levels of drag generating swirling exhaust flows, (2) identify noise mechanisms and dissect source locations in such flows, and (3) establish scaling laws and the relation between swirl, drag and radiated sound power. The working hypothesis is that the acoustic signature is comprised of quadrupole-type turbulent mixing noise of the swirling core flow and scattering noise from vane boundary layers and turbulent eddies of the burst vortex structure by sharp edges such as the exposed trailing edge of the nacelle, centerbody and the pylon.

The paper is organized as follows. First, an overview of the relevant aerodynamic flow features of the investigated swirling exhaust flows is given. Next, plausibility arguments for the qualitative assessment of the acoustic sources are followed by the design of acoustic experiments. Then, the measured far-field spectra are analyzed and key acoustic features are identified. Using DAMAS array data processing noise source maps are established and are used to investigate the noise generation mechanisms. The source noise in different regions of the flow field is quantified. Both stable swirling flows and swirling flows with vortex breakdown are considered. Then, scaling laws are established using the source noise maps, corroborating the hypotheses and qualitative assessments. Last, the work is summarized and concluding remarks are given. 


\section{Aerodynamic Features of Swirling Exhaust Flows}

The aerodynamic design and analysis of the ram-pressure driven nacelle with stationary swirl vanes is discussed in detail in Shah et al. ${ }^{2}$ and only the relevant features of the swirling flow are briefly summarized here. The vanes are designed to yield a Burger vortex-type circulation distribution approaching forced vortex flow in the core and free vortex type behavior on the outer periphery. A model scale engine nacelle with stationary swirl vanes of outer diameter $0.179 \mathrm{~m}$ was built and tested in the MIT Wright Brothers Wind Tunnel. The vanes are integral to a disk which can be exchanged to achieve various swirl angle settings. Swirl angle settings of 0 , 34, 41, 47, 53, 57 and 64 degrees were implemented and experimentally tested using hot-wire anemometry and flow visualization. Figure 3 shows a comparison between the
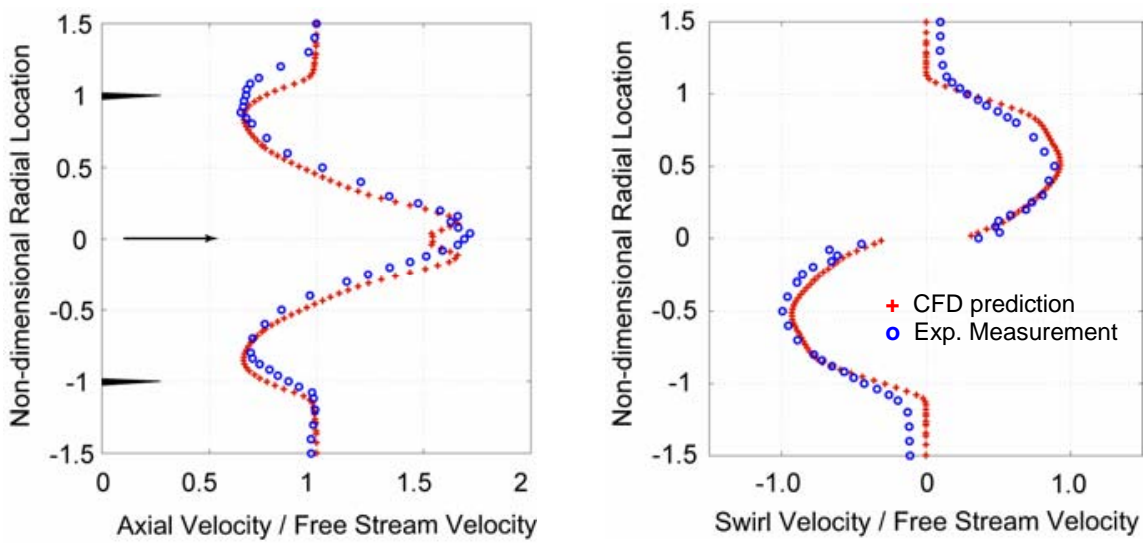

Figure 3. Experimental validation of predicted axial and tangential velocity profiles at two diameters downstream of nacelle for swirl vane setting of $47^{\circ}$ : (+) CFD prediction, (o) hot-wire measurements.

predicted axial and tangential velocity profiles using three-dimensional steady RANS calculations (marked by red pluses) and hot-wire traverse measurements (marked by blue circles) for the nacelle with a swirl vane setting of 47 degrees. The results indicate good agreement between the predictions and the experiment validating the swirl vane design. Note that there is a region of increased axial velocity and high shear near the core region and a deficit in axial velocity leading to a wake like structure on the outer periphery. The excess in centerline axial velocity is correlated with both the swirl angle setting and the centerline pressure defect.

The swirl vane angle setting of 47 degrees yields an experimentally measured drag coefficient of 0.83 , the highest drag coefficient without vortex breakdown. The predicted drag of 0.8 falls a little short of the measurement which is suggested to be due to the slight under-turning of the flow as seen in Figure 3 on the right. The limiting phenomenon for stable flow is vortex breakdown which is associated with the rapid expansion of the core when a critical swirl angle distribution is reached ${ }^{1-3}$. For the Burger vortex distribution implemented here, vortex breakdown was measured to occur for swirl vane angle settings greater than 50 degrees in good agreement with the theory and simulations. A separation bubble forms downstream of the nacelle and creates blockage and high levels of turbulence, reducing the drag generation capability and dramatically increasing the noise level as shown in Figure 2. The mechanism of noise generation is the focus of this paper and is discussed next in-depth.

\section{Qualitative Description and Plausibility Arguments for Acoustic Sources}

It is hypothesized that the acoustic signature is comprised of quadrupole-type turbulent mixing noise of
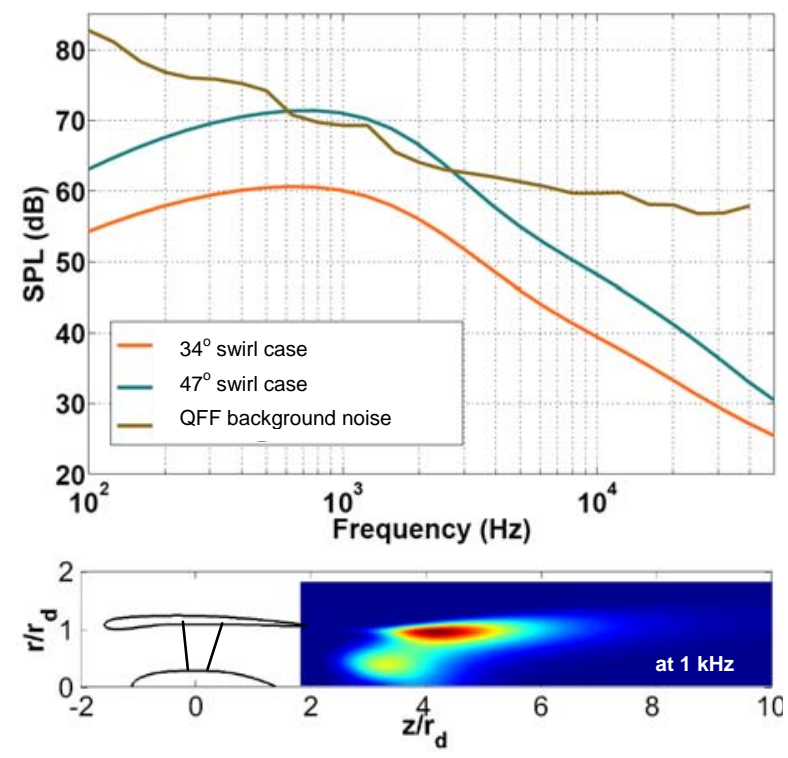

Figure 4. Predicted fine-scale turbulence noise of swirling exhaust flow at polar angle of $90^{\circ}$ and distribution of spectral density integrand.

the swirling core flow and scattering noise from vane boundary layers and turbulent eddies of the burst vortex structure near sharp edges exposed to the flow. In what follows, a qualitative description and plausibility arguments 
for the acoustic sources are given in support of the experimental noise source measurements. In particular, steady axisymmetric RANS simulations of the swirling exhaust flow are coupled with an acoustic analysis based on the fine-scale turbulence jet noise model by Tam et al. ${ }^{8}$ and the description for swirling sources by Tanna ${ }^{5}$. Scaling laws for scattering of aerodynamic sound by sharp edges based on Lighthill's acoustic analogy and using the reciprocal theorem $^{9,10}$ are employed to support the hypothesis that scattering noise is the dominant mechanism for noise generation.

In the absence of a rigorous theoretical framework to predict the quadrupole noise from a swirling flow it is assumed that the source strength between helically and axially convected sources is unchanged. The fine-scale turbulence mixing noise of the swirling flow at a polar angle of 90 degrees is estimated from steady axisymmetric RANS calculations in combination with Tam's semi-empirical expression for the spectral density $S$ of straight jets,

$$
S(\vec{x}, \omega)=4 \pi\left(\frac{\pi}{\ln 2}\right)^{3 / 2} \times \iiint_{V o l} \frac{\hat{q}_{s}^{2} l_{s}^{3}}{c_{o}^{2} \tau_{s}} \frac{\exp \left[\frac{\omega^{2} l_{s}^{3}}{\bar{u}^{2}(4 \ln 2)}\right]}{\left[1+\omega^{2} \tau_{s}^{2}\left(1-\frac{\bar{u}}{a_{\infty}} \cos \theta\right)^{2}\right]}\left|p_{a}\left(\vec{x}_{2}, \vec{x}, \omega\right)\right| d \vec{x}_{2}
$$

In the above expression, the averaged turbulence kinetic energy $k$ and dissipation rate $\varepsilon$ from the simulation are used in combination with the empirically defined constants by Tam et al. ${ }^{8}$ to set the turbulence decay time $\tau_{s}$, length scale $\ell_{\mathrm{s}}$ and the RMS of the fluctuating kinetic energy of the fine-scale turbulence $\hat{q}_{\mathrm{s}}$. The spectral density is further modified by a noise amplification factor for the helical motion of sources given by Tanna ${ }^{5}$

$$
\frac{\left\langle p^{\prime 2}\right\rangle_{\text {Helical }}}{\left\langle p^{\prime 2}\right\rangle_{M_{\theta}=0}}=F_{1}\left(\alpha^{\prime}\right)+\left(\frac{\Omega}{v}\right)^{2} F_{2}\left(\alpha^{\prime}\right)+\left(\frac{\Omega}{v}\right)^{4} F_{3}\left(\alpha^{\prime}\right) \quad \text { with } \quad \alpha^{\prime}=\frac{M_{\theta} \sin \psi}{1-M_{\theta} \sin \psi},
$$

where $v$ is the source radian frequency, $\Omega$ is the rotation radian frequency, $F_{1}, F_{2}$ and $F_{3}$ are functions, and $\alpha$ ' is defined in terms of the observer angle $\psi$ and the axial and tangential Mach numbers of the swirling flow. The 1/3 octave band sound pressure spectrum may then be written as

$$
S P L(f)=10 \log \left[\frac{\left\langle p^{\prime 2}\right\rangle_{\text {Helical }}}{\left\langle p^{\prime 2}\right\rangle_{M_{\theta}=0}} \frac{4 \pi S(\vec{x}, \omega)}{p_{\text {ref }}^{2}}\right]+10 \log \left(\Delta f_{H z}\right)
$$

In the limit of zero swirl angle a straight jet or a wake flow is recovered. The developed methodology was validated against jet noise data available in literature. The methodology was subsequently used to estimate the finescale turbulence mixing noise of the swirling exhaust flows under consideration. Figure 4 on the bottom shows the distribution of the spectral density integrand of Equation 1 and the predicted 1/3 octave frequency spectra for swirl vane angles settings of 34 and 47 degrees together with the measured background noise spectrum of the NASA Langley Quiet Flow Facility (QFF). The results indicate that the estimated noise sources seem to be concentrated in the shear layer and near the core of the swirling flow. The fine-scale turbulence mixing noise is rather quiet as compared to the background noise. The qualitative assessment suggests that: (1) other noise sources that are stronger radiators than the quadrupoles of the turbulence mixing noise might be at play and need to be captured, (2) swirlingflow turbulence mixing noise increases with the level of swirl, and (3) from a measurement perspective, phased microphone array methods together with advanced source mapping algorithms need to be employed to dissect the noise generation mechanisms. It will be shown that, quadrupole-type turbulence mixing noise and over most of the frequency range, scattering noise is the dominant noise source.

The scattering of aerodynamic sound by a sharp edge, such as the trailing edge of the nacelle, the centerbody or the pylon can be described using Lighthill's acoustic analogy and by applying the reciprocal theorem ${ }^{9-10}$. The scaling of the amplitude of density perturbations generated by turbulent quadrupoles near a scattering surface can be expressed as

$$
\left\langle\rho^{\prime 2}\right\rangle \propto \rho_{o}^{2} M^{5}\left(\frac{l}{y}\right)^{3} \frac{l^{2}}{r^{2}} \sin ^{2}\left(\frac{1}{2} \theta\right)
$$


where $l$ is the length scale of the turbulent eddy located at a distance $y$ from the edge. At low Mach number the generated scattering sound is more than the free space sound of the same eddy by a factor $M^{-3} l^{3} / y^{3}$. Scattering surfaces dominate the source structure of low Mach number flows and it will be shown that the acoustic signature of the drag generating swirling exhaust flows is strongly governed by scattering noise from vane boundary layers and turbulent structures in the vortex core by sharp edges. The increased noise levels for vortex breakdown, as shown in Figure 2, are due to the combined effect of a reduced distance between the eddies of the burst vortex structure and the solid edges, and an increase in the turbulence intensity and the spatial extent thereof.

\section{Design of Experiments and Acoustic Testing}

The scale model nacelle with various swirl vane setting angles was tested in the NASA Langley Quiet Flow Facility (QFF). The facility has been used in measurements of airfoil self-noise ${ }^{11}$, distributed trailing edge noise ${ }^{12}$, flap side edge noise $e^{13}$, and leading edge slat noise ${ }^{14}$. The $9.1 \mathrm{~m}$ by $6.1 \mathrm{~m}$ by 7.6 $\mathrm{m}$ anechoic test chamber has a vertically flowing $0.6 \mathrm{~m}$ by $0.9 \mathrm{~m}$ free jet exhausting through the ceiling. Free jet Mach numbers of up to 0.17 can be achieved. A total of 6 fixed-pole microphones are located on either side of the free jet and a Medium Aperture Directional Array (MADA) consisting of 41 microphones is mounted on a boom that can rotate on a polar arc as shown in Figure 5 on the left. The nacelle was mounted on a pylon with a NACA 0022 profile that was attached
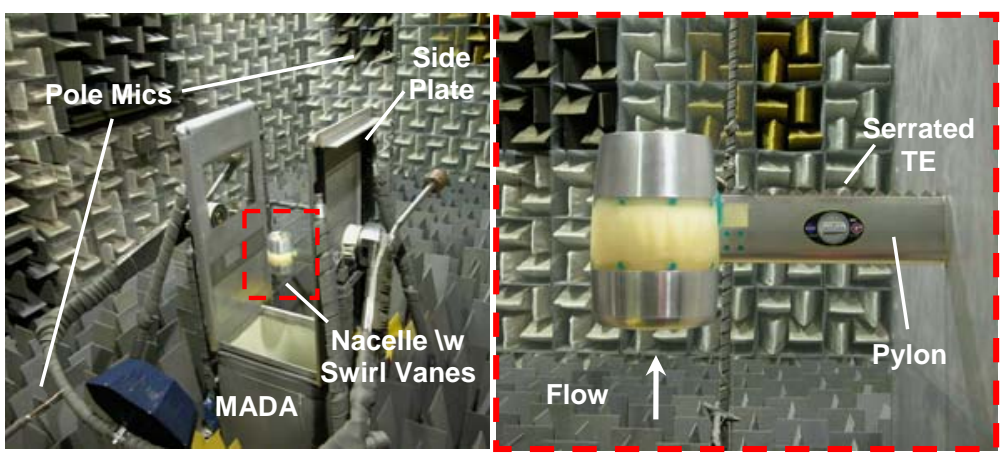

Figure 5. Scale model nacelle with swirl vanes mounted in the QFF test facility at NASA Langley. to one of the two side plates of the wind tunnel. The model size was chosen such that, given the spatial test window, the swirling exhaust flow stayed well within the potential core of the free jet. To ensure the noise signature is not affected by possible interactions between the shear layer of the free jet and the swirling exhaust flow, the nacelle was mounted at different axial locations. Noise measurements showed that the acoustic signature was insensitive to the nacelle location. The boundary layers of the pylon, the nacelle and the swirl vanes were tripped to ensure turbulent flow. The pylon trailing edge was serrated to mitigate trailing edge noise that could contaminate the swirling exhaust flow noise measurements.

The experimental test matrix was composed of pylon only measurements, empty nacelle tests, and measurements with swirl vane angle settings of $0,34,41,47,53,57$ and 64 degrees, ranging from zero swirl to highly swirling flow with vortex breakdown. In addition, tests were conducted with positive and negative nacelle angles of attack and various upstream flow non-uniformities. This included a blade wake generator simulating an upstream blade row and perforated plates of 120 degrees circumferential extent modeling inlet flow distortion common in engine inlets..

The Deconvolution Approach for Mapping of Acoustic Sources (DAMAS) developed by Brooks and Humphreys ${ }^{15,16}$ was employed to map the noise sources of the drag generating swirling exhaust flows. In previous research, the technique has been successfully applied to distributed sources near solid bodies such as wing trailing edges, leading edge slats or flap side edges. This is the first time that the DAMAS technique is applied to measure quadrupole-type volume sources.

\section{Swirling Exhaust Flow Sound Pressure Spectra}

To help identify scaling laws and directivity, microphone array data was acquired at wind tunnel Mach numbers of $0.07,0.09,0.11,0.13,0.15$ and 0.17 , and at various MADA array angles. Two forward angles at -124 and -107 degrees, the sideline angle at -90 degrees, and three aft angles at $-73,-56$ and -45 degrees were investigated. Figure 6 illustrates the acoustic features of various swirling exhaust flows together with the wind tunnel only and the empty nacelle (no swirl vanes) noise. $17.44 \mathrm{~Hz}$ narrowband auto-spectra at a polar angle of -90 degrees and an observer distance of $1.5 \mathrm{~m}$ are plotted for a wind tunnel Mach number of 0.17 . All spectra are primarily broadband in nature, with good signal-to-noise ratio for frequencies above $1 \mathrm{kHz}$. Trailing edge noise due to the scattering of turbulent boundary layer structures is observed in the $2-3 \mathrm{kHz}$ range for the pylon and nacelle only cases. It will be shown in 
the next section that the sound pressure levels scale with the free stream Mach number to a power between 5 and 5.5, consistent with classic airfoil self-noise measurements ${ }^{11}$.

The sound pressure spectra of swirling flows may be classified into two families: (i) stable swirling flows with broadband peaks in the lower frequency range (swirl vane angle settings 0 to 47 degrees), and (ii) flows with vortex breakdown (swirl vane angle settings 53 to 64 degrees) exhibiting white noise like spectra dramatically louder than their stable counterparts. The broadening of the low frequency peak in the 47 degree vane angle setting case suggests transition from stable exit flow to vortex breakdown, conjectured to be due to the onset of standing waves patterns on the vortex core (for details see Shah et al. ${ }^{2}$ ).

Comparison of the nacelle only case with the stable swirling flow cases (0 through 47 degrees vane angle setting) suggests that the presence of the swirl vanes gives rise to low frequency broadband peaks seen in the $5-10 \mathrm{kHz}$ range. The spectra roll off at higher frequencies and the noise levels and peak frequencies increase with swirl angle setting. These observations lead to the following hypotheses:

(1) The broadband spectral peaks of the stable swirling flow cases are suggested to be due to the scattering noise of vane boundary layers governed by the swirling exhaust flow Mach number.

(2) The higher frequency noise is predominantly due to quadrupole-type mixing noise of swirling exhaust flow.

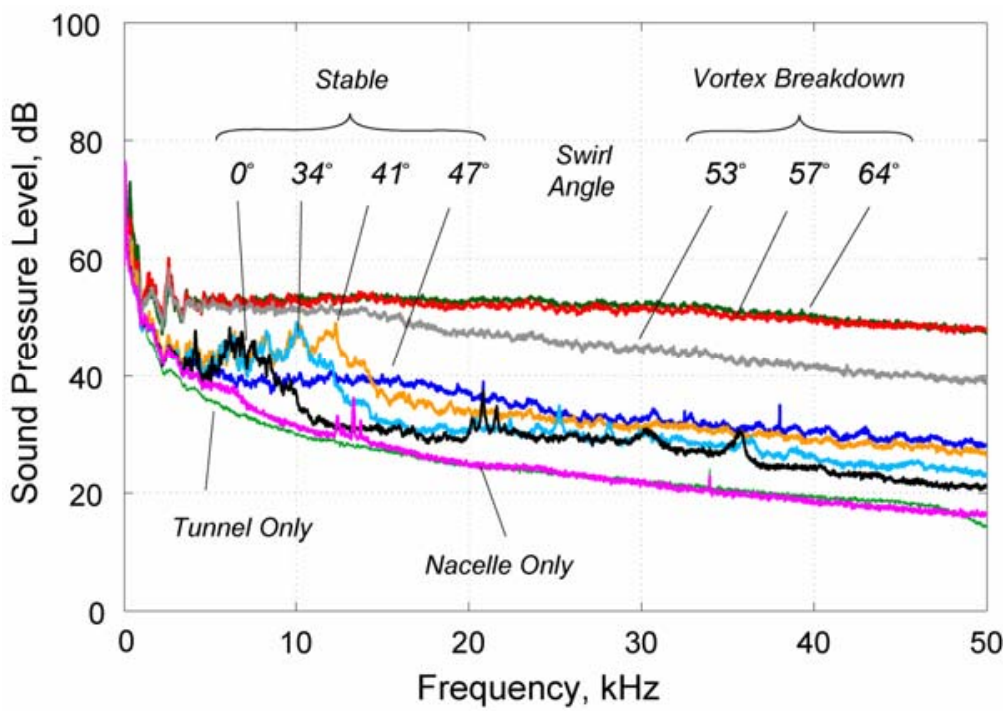

Figure 6. Narrowband sound pressure spectra for swirling exhaust flows at $M=0.17$, polar angle -90 deg and $1.5 \mathrm{~m}$ distance.

(3) The vortex breakdown spectra are dominated by scattering noise of the turbulent eddies of the burst vortex structure near sharp edges and turbulent flow mixing noise, suggesting a Mach number scaling power between five and eight.

These hypotheses are assessed in the next section by dissecting the overall noise signature into zonal spectra using DAMAS post-processing and by extracting Mach number scaling laws to determine the fundamental noise mechanisms. Assuming that the swirling flow Mach number is found to be the governing parameter for scattering type noise at a fixed swirl angle setting, the nacelle exit zonal spectra at different free stream Mach numbers should collapse to a single spectrum using a power law for the sound pressure level and peak frequency scaling based on Strouhal number. A similar approach is taken to assess quardupole-type turbulent mixing noise and vortex breakdown noise.

\section{Dissection of Noise Sources and Scaling Laws}

Two specific cases, one for stably swirling flow at the highest drag coefficient and the other case for vortex breakdown, are first discussed in detail to demonstrate the dissection of noise sources using DAMAS postprocessing. Next, the scaling laws are established using the entire test matrix.

The nacelle with a swirl vane angle setting of 47 degrees was tested at various polar angles and Mach numbers. This swirl case exhibits stable flow as discussed earlier and illustrated by flow visualization in Figure 7. Helical motion of fluid particles is observed and viscous diffusion and mixing of the swirling exhaust flow occurs downstream of the nacelle. Model scale 1/3 octave-band auto-spectra of two array microphones are also plotted in Figure 7 marked by the light blue and magenta curves for a tunnel Mach number of 0.17 and a boom angle of -90 degrees (sideline position similar to the one shown in Figure 5 on the left). The QFF empty tunnel background noise 

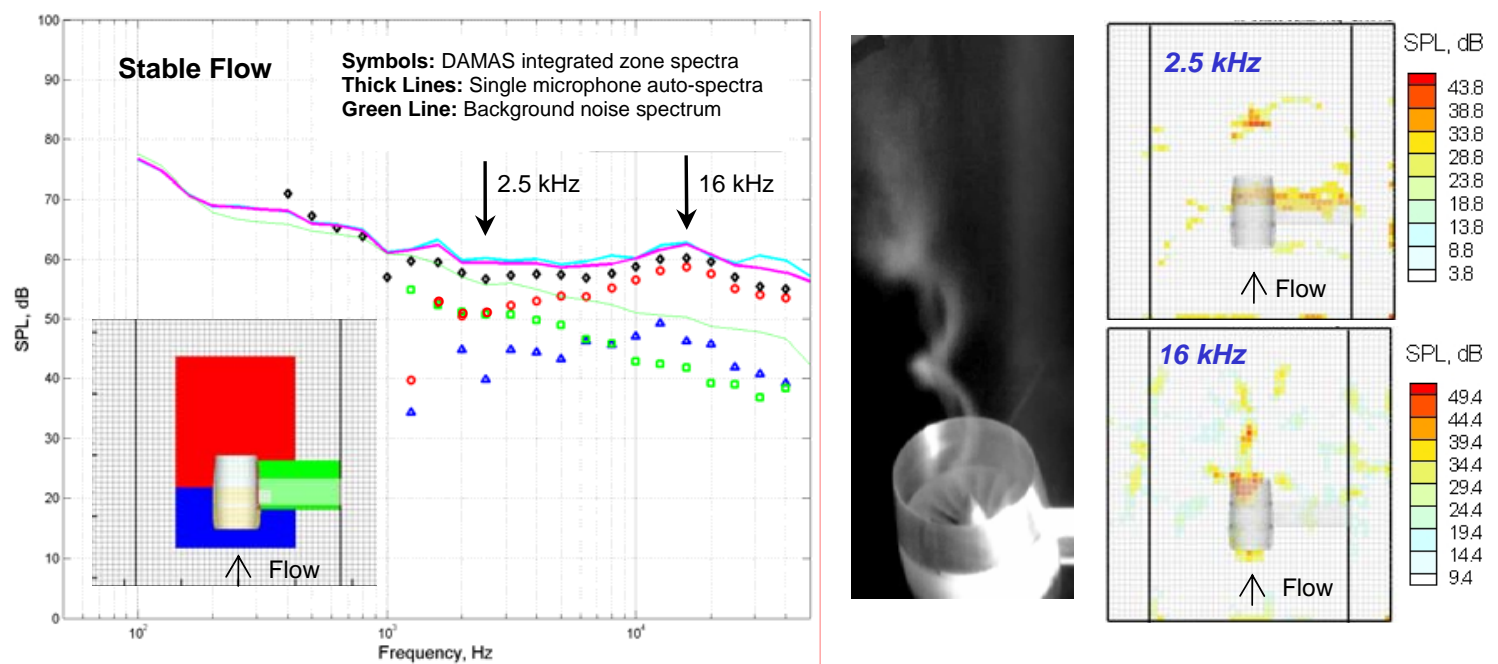

Figure 7. 1/3 octave band spectra and DAMAS source maps for swirl vane angle setting of $\mathbf{4 7}^{\circ}$.

is shown as the thin green line indicating again that data is acquired with a high signal-to-noise ratio for frequencies greater than $1 \mathrm{kHz}$. The swirling flow spectra are broadband with slightly pronounced activity near $2.5 \mathrm{kHz}$ and 16 $\mathrm{kHz}$ as indicated by the arrows.

\section{A. DAMAS Noise Source Mapping and Zonal Spectra}

The DAMAS post-processing allows the spatial mapping of sources. For any given frequency the total sound pressure level of a zone on the scanning grid can be obtained by simply summing the mean square value of the pressure fluctuations of the grid points of interest. In Figure 7, the following three zones are defined: the red zone is the aft region, enclosing the swirling flow and the nacelle exit, the blue zone is the forward region enclosing the nacelle inlet, and the green zone is the pylon region containing the pylon leading and trailing edge. To assess this procedure the three zones are summed up at each of the 1/3 octave band frequencies (black diamonds) and compared against the single microphone auto-spectra. Good agreement is achieved validating the zonal mapping procedure. Note that the sum of the zonal spectra is slightly below the single micro-phone auto-spectra since the former is limited to the zones only and thus does not contain all the noise sources in the field. Using the same color code, each individual zonal spectrum is also shown in Figure 7. The results indicate that the pylon noise (green squares) is well below the background noise and most dominant in the 1 to $3 \mathrm{kHz}$ range. This is in agreement with the source noise map at $2.5 \mathrm{kHz}$ shown on the top right. The noise radiated from sources in the forward zone is at much lower sound pressure levels as seen by the blue triangles. Sound from the aft zone (red circles) dominates the acoustic signature
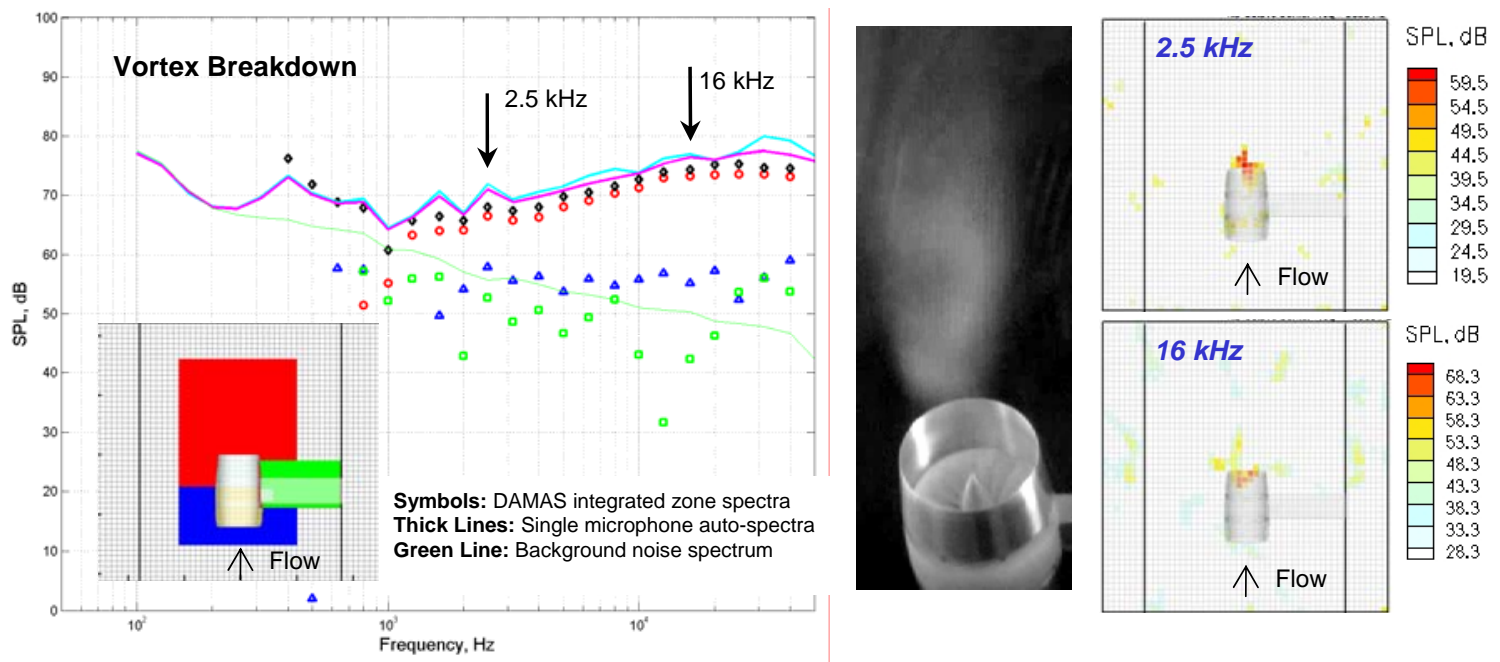

Figure 8. 1/3 octave band spectra and DAMAS source maps for swirl vane angle setting of $57^{\circ}$. 
for frequencies greater than $3 \mathrm{kHz}$. There are volume sources downstream of the nacelle trailing edge in the vortex core visible in the source noise maps at 2.5 and $16 \mathrm{kHz}$. As discussed next, the Mach number scaling of the frequency spectra shows that the very high-frequency noise is of quadrupole type. Scattering noise near the nacelle trailing edge and centerbody is also observed. It will be shown that the sound pressure level scales with the Mach number close to a power of 5 , corroborating the hypothesis that this is the dominant noise generation mechanism.

Figure 8 depicts similar plots for a swirl vane angle setting of 57 degrees for which vortex breakdown occurs. The flow visualization illustrates bubble-type vortex breakdown ${ }^{2}$ near the nacelle trailing edge. The frequency spectrum indicates dominant acoustic activity in the aft region for all selected frequency bands. An increase in noise levels of 10 to $15 \mathrm{~dB}$ as compared to the stable flow case shown in Figure 7 is observed. The source noise maps corroborate the hypothesis that scattering noise from the nacelle and centerbody trailing edge and turbulent mixing noise dominate the acoustic signature.

\section{B. Scaling Laws for Swirling Exhaust Flow Noise}

To further assess the nature of the noise sources, Strouhal number and Mach number based scaling of zonal spectra is carried out using the measurements at different tunnel velocities and different swirl vane angle settings. The spectra of the stable swirling flow cases are found to scale differently at low and high frequency, indicating that multiple noise source mechanisms are at play. For a given swirl vane angle setting the characteristic Mach number is found to be the free-stream Mach number, while across swirl vane angle settings the characteristic Mach number is found to be a representative vortex core Mach number. The vortex breakdown cases exhibit a markedly different acoustic signature and appear to scale according to a combination of scattering and quadrupole-type sources concentrated near the nacelle exit.

\begin{tabular}{|c|c|c|c|}
\hline \multirow{2}{*}{$\begin{array}{c}\text { Swirl Vane } \\
\text { Angle Setting }\end{array}$} & \multicolumn{3}{|c|}{ Deduced Power Law (n) } \\
\cline { 2 - 4 } & $\begin{array}{c}\text { Exit } \\
\text { Domain }\end{array}$ & $\begin{array}{c}\text { Outflow } \\
\text { Domain }\end{array}$ & $\begin{array}{c}\text { Entire } \\
\text { Aft Region }\end{array}$ \\
\hline Empty Nacelle & $5-5.5$ & - & - \\
\hline 0 deg & $5-5.5$ & - & - \\
\hline 34 deg & $\sim 6.5$ & $\sim 8.0$ & - \\
\hline 41 deg & $\sim 6.5$ & $\sim 8.0$ & - \\
\hline 47 deg & $\sim 6.5$ & $\sim 8.0$ & - \\
\hline 53 deg & - & - & 7.5 \\
\hline 57 deg & - & - & 7.5 \\
\hline 64 deg & - & - & 7.5 \\
\hline
\end{tabular}

Table 1. Mach number based power law exponents for zonal spectra defined in Figure 9.

The first step in the dissection of the noise sources is the scaling of zonal spectra. In general, the entire region aft of the swirl tube nacelle exit was found to be the

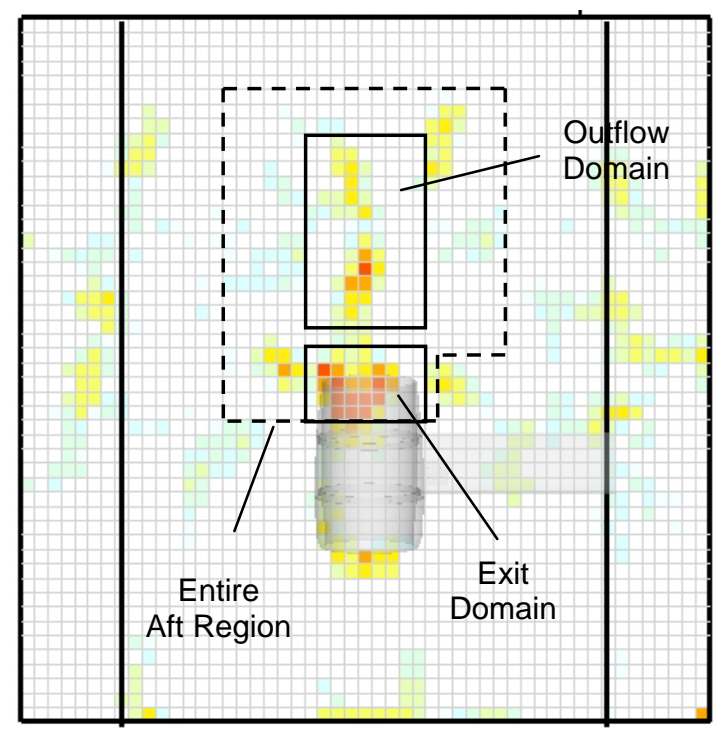

Figure 9. Definition of sub-domains near nacelle exit for DAMAS based identification of noise source mechanisms.

major contributor to the overall noise signature. This region was then further divided into the two sub-domains shown in Figure 9, the nacelle exit domain encompassing regions close to the nacelle trailing edge, and the swirling outflow domain downstream of the exhaust plane.

Table 1 summarizes the experimentally determined power law exponents using Mach number scaling of DAMAS integrated zonal spectra of the different test articles. The following conclusions may be drawn from the table regarding the nature of the noise sources. To begin, we consider the working hypothesis that the sound generated near the nacelle exit is primarily due to vane and nacelle trailing edge scattering noise. The deduced Mach number exponent of 5 to 5.5 in the empty nacelle and no swirl (0 degree swirl vane angle setting) suggests that the DAMAS system is well calibrated to detect airfoil self-noise type sources. Figure 10 depicts the spectra for 

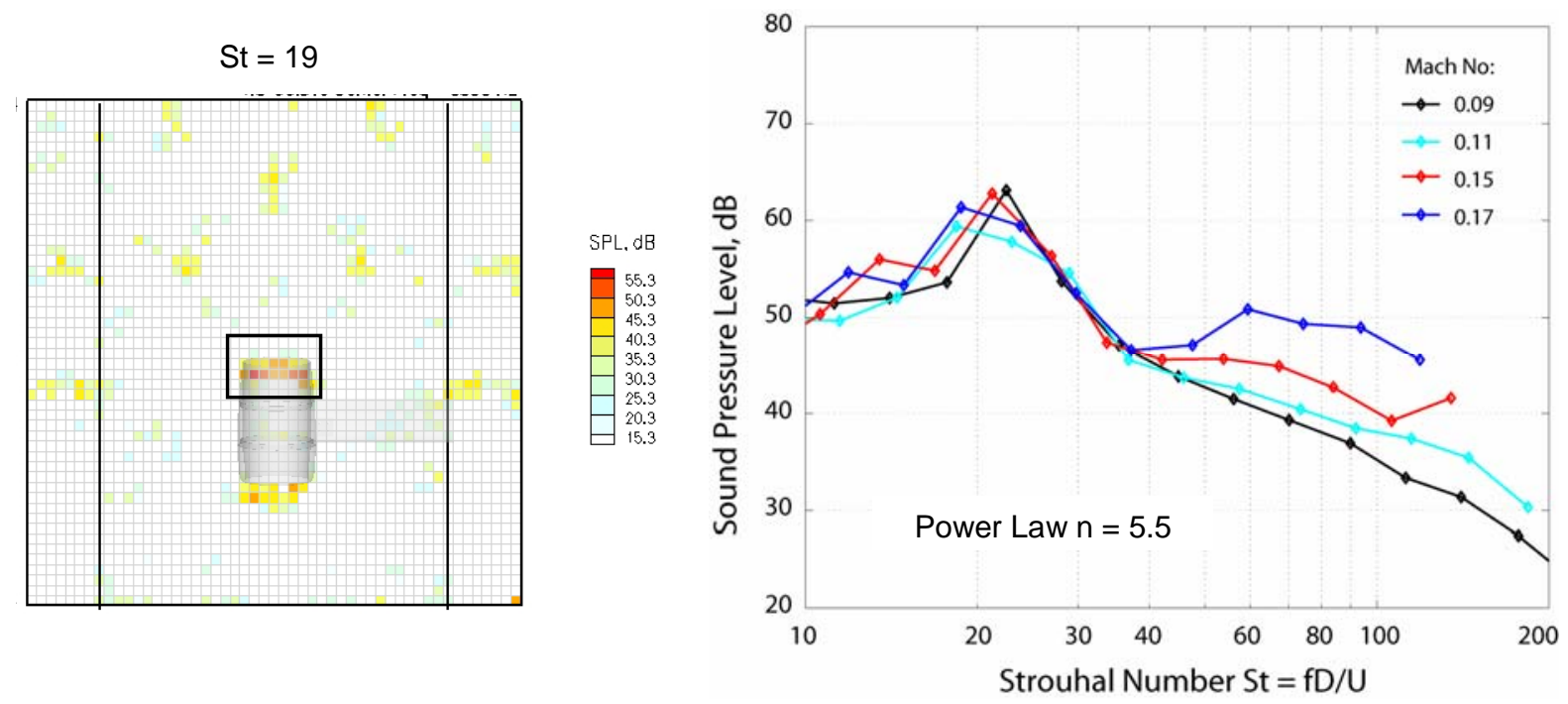

Figure 10. Exit domain based 1/3 octave band spectra scaled with Mach number to power 5.5 for 0 degree swirl vane angle setting (no swirl); DAMAS source noise map at $\mathrm{St}=19$.

a swirl vane setting of 0 degrees at various Mach numbers, scaled at a power of 5.5. The Strouhal number is based on nacelle diameter.

Stable Swirling Flow Noise. The zonal spectra for all vane angle settings that yield stable swirling flow were analyzed and only the case for a swirl angle setting of 47 degrees is discussed here. The results are summarized in Table 1 and suggest that the noise sources are comprised of two distinct mechanisms present in the two sub-domains of the nacelle aft region. Near the nacelle exit the zonal spectra scale with a power of 6.5 as depicted in Figure 11 for a swirl vane angle setting of 47 degrees. The broadband frequency peaks identified earlier in Figure 6 correspond to a Strouhal number of 48 and are conjectured to be due to scattering and dipole type noise sources as shown by the DAMAS source noise map in Figure 11. While scattering and dipole type noise effects are present near the exit of the nacelle, the analysis together with the plausibility arguments given earlier suggest that the swirling outflow domain is dominated by quadrupole-type turbulent mixing noise. In Figure 12 the 1/3 octave band
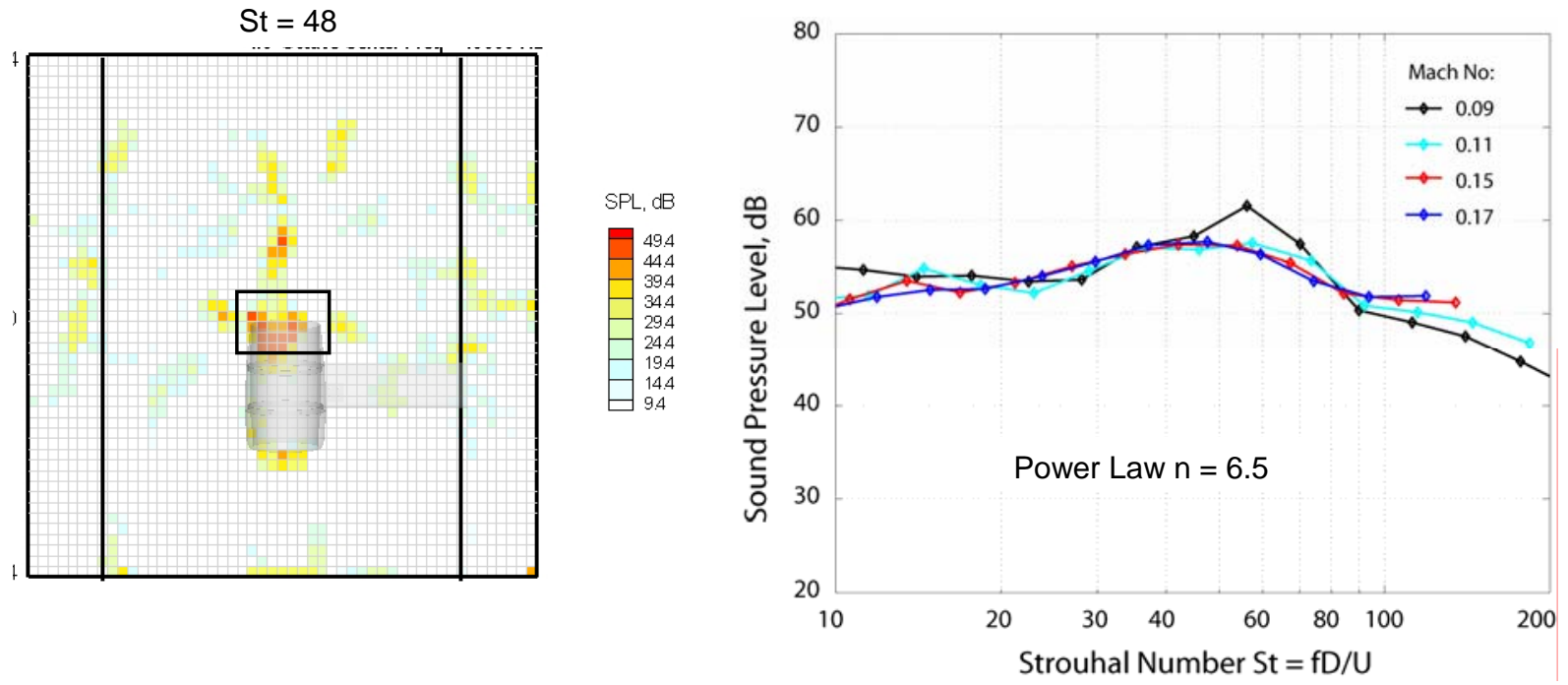

Figure 11. Exit domain based 1/3 octave band spectra scaled with Mach number to power 6.5 for 47 degree swirl vane angle setting; DAMAS source noise map at $\mathrm{St}=48$. 

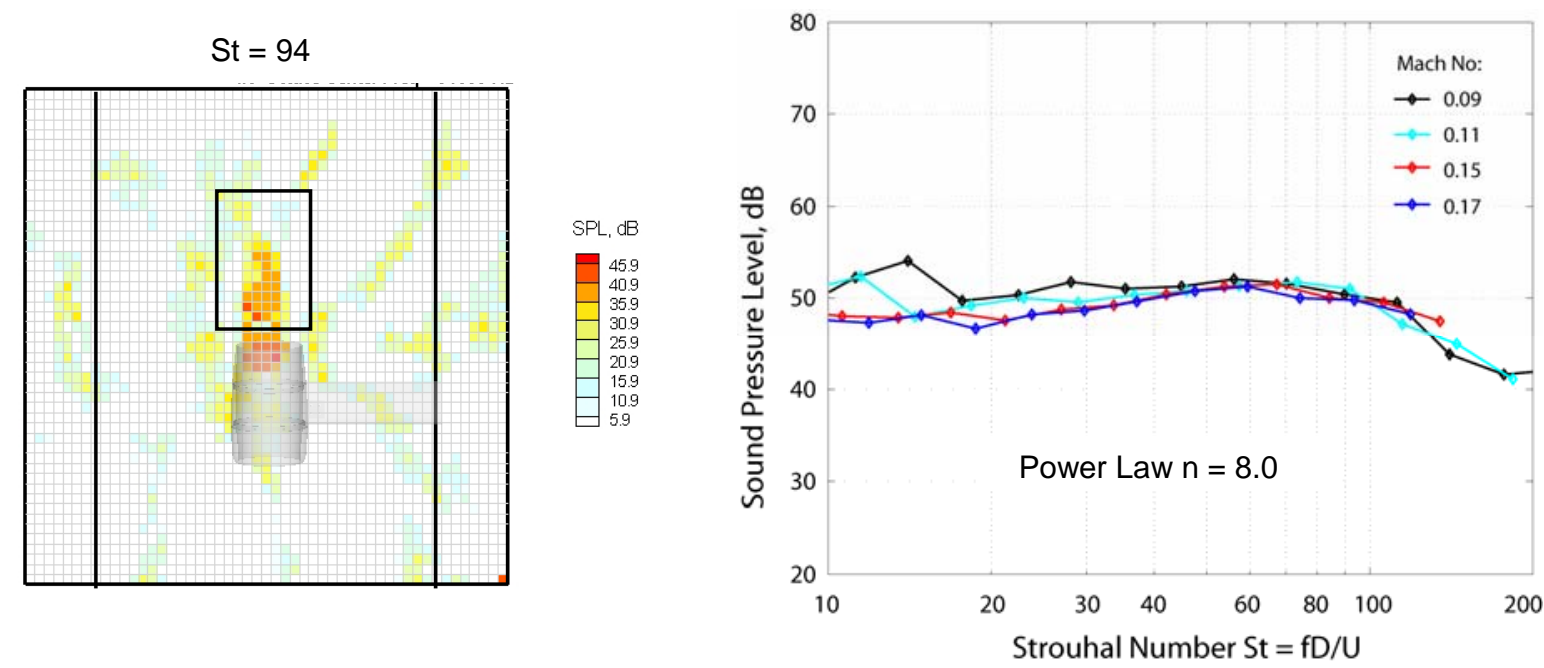

Figure 12. Outflow domain based $1 / 3$ octave band spectra scaled with Mach number to power 8.0 for 47 degree swirl vane angle setting; DAMAS source noise map at $\mathrm{St}=\mathbf{9 4}$.

spectra are scaled with the $8^{\text {th }}$ power of free-stream Mach number supporting the hypothesis. It is worth noting that these experiments constitute the first application of the DAMAS post-processing technique to volume sources, successfully demonstrating the identification of quadrupole sources and making the procedure also viable for jetnoise diagnostics.

Vortex Breakdown Noise. The vortex breakdown noise was audibly louder, resembling white noise with a "crackle" like sound compared to the "hiss" like noise of the stable swirling cases. As illustrated by the flow visualizations presented earlier in the paper, swirl angle settings in excess of 47 degrees yield classic bubble-type vortex breakdown near the nacelle trailing edge, with dominant acoustic activity in the aft region for all frequency bands. Noise appears to be intensified by the turbulence of the burst vortex structure scattered near the nacelle and center-body trailing edges. Similar Mach number scaling performed on the zonal spectra for swirl angle settings of 53, 57 and 64 degrees shows excellent agreement for a derived power of 7.5, over the entire aft zone. The results indicate that both scattering and turbulent mixing noise are at play. Figure 13 depicts the scaled spectra and a typical DAMAS source map at a Strouhal number of 94 illustrating that the burst vortex structure close to the duct exit concentrates the noise source in that region.

In summary, three distinct acoustic features associated with stable swirling flow and vortex breakdown in the vicinity of nacelle and vane edges were identified. The results suggest that stable swirling flow noise is governed by two distinct source mechanisms: scattering and dipole type noise is observed to scale with the freestream Mach number to the power 6.5 for Strouhal numbers near 50, and quadrupoletype turbulent mixing noise scales with a power of 8 for a Strouhal number near 100 . Swirling flows with vortex breakdown have a substantially higher overall noise levels

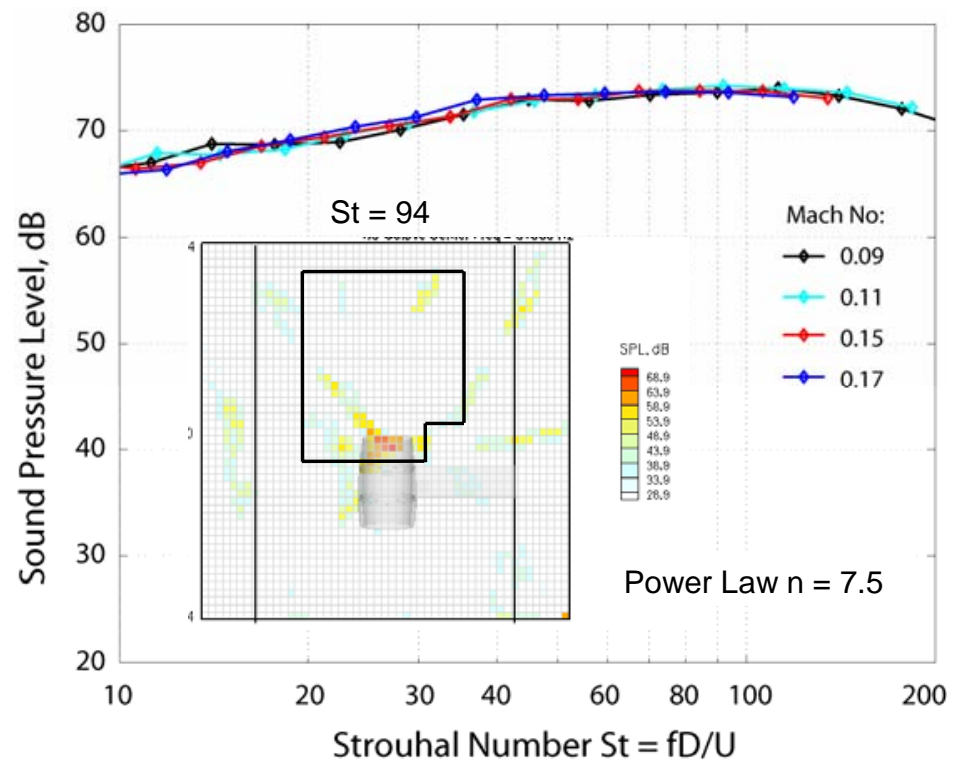

Figure 13. Aft region based 1/3 octave band spectra scaled with Mach number to power 7.5 for 57 degree swirl vane angle setting; DAMAS source noise map at $\mathrm{St}=\mathbf{9 4}$. 
and appear to scale with free-stream Mach number to the power of 7.5 for the entire Strouhal number range. This suggests that the vortex breakdown noise generation process is governed by both scattering and quadrupole-type sources.

\section{Summary and Conclusions}

A rigorous aero-acoustic assessment of drag generating swirling exhaust flows was presented. The technical approach combined an in-depth aerodynamic analysis, plausibility arguments to qualitatively describe the nature of acoustic sources, and detailed, quantitative acoustic measurements using a medium aperture directional microphone array in combination with a previously established Deconvolution Approach for Mapping of Acoustic Sources (DAMAS). A model scale engine nacelle with stationary swirl vanes was designed and tested in the NASA Langley Quiet Flow Facility at a full-scale approach Mach number of 0.17 . The analysis showed that the acoustic signature is comprised of quadrupole-type turbulent mixing noise of the swirling core flow and scattering noise from vane boundary layers and turbulent eddies of the burst vortex structure near sharp edges. For the highest stable swirl angle setting of 47 degrees, a nacelle area based drag coefficient of 0.8 was achieved with a full-scale OASPL of about $40 \mathrm{dBA}$ at the ICAO approach certification point.

\section{Acknowledgments}

The authors would like to thank Professors Jack Kerrebrock and Ed Greitzer for their inspiration and insightful discussions on swirling flows. The invaluable help and support of Dan Stead, Larry Becker, Jay Moen and Dennis Kuchta at NASA Langley is gratefully acknowledged. Special thanks go to Ronnie Geouge for his assistance with the test hardware throughout the experiments. This research was funded by the NASA Langley Research Center, Dr. Russell Thomas contract monitor.

\section{References}

1. Shah, P., "Novel Turbomachinery Concepts for Highly Integrated Airframe / Propulsion Systems,” Ph.D. thesis, Department of Mechanical Engineering, MIT, October 2006.

2. Shah, P., Mobed, D., and Spakovszky, Z., “A Novel Turbomachinery Air-Brake Concept for Quiet Aircraft,” in ASME IGTI Turbo Expo, paper GT2007-27635, May 14-17, Montreal, Canada, 2007.

3. Greitzer, E., Tan, C., and Graf, M., "Internal Flow - Concepts and Applications, “ Cambridge University Press, 2004.

4. Tanna, H., and Morfey, C., "Sound Radiation from Point Sources in Circular Motion”, Journal of Sound and Vibration, Vol. 16(3), pp. 337 - 348, 1971.

5. Tanna, H., "On the Effect of Swirling Motion of Sources on Subsonic Jet Noise,” Journal of Sound and Vibration, Vol. 29(3), pp. $281-293,1973$.

6. Schwartz, I., "Swirling-flow Jet Noise Suppressors for Aircraft Engines," AIAA paper 1973-1003, presented at the AIAA Aero-Acoustics Conference, Seattle, WA, Oct. 15-17, 1973.

7. Lu, H., Ramsay, J., and Miller, D., “Noise of Swirling Exhaust Jets,” AIAA Journal, Vol. 15 No. 5, pp. 642 - 646, May, 1977.

8. Tam, C., and Auriault, L., “Jet Mixing Noise from Fine-Scale Turbulence,” AIAA Journal, Vol. 37 No. 2 , pp. 145 - 153 , February, 1999.

9. Ffowcs Williams, J., and Hall, L., "Aerodynamic sound generation by turbulent flow in the vicinity of a scattering half plane,” J. of Fluid Mechanics, Vol. 40 Part 4, pp. 657 - 670, 1970.

10. Dowling, A., and Ffowcs Williams, J., "Sound and Sources of Sound,” Ellis Horwood Limited, 1983.

11. Brooks, T., Pope, S., and Marcolini, M., “Airfoil Self-Noise and Prediction,” NASA Reference Publication, 1218, July, 1989.

12. Hutcheson, F., and Brooks, T., "Measurement of trailing edge noise using directional array and coherent output power methods,” International Journal of Aeroacoustics, 1 (4), pp. 329 - 354, 2002.

13. Brooks, T. and W. Humphreys Jr., "Flap Edge Aeroacoustic Measurements and Predictions,” Journal of Sound and Vibration, Vol. 261, pp. 31 - 74, 2003.

14. Mendoza, J., Brooks, T. and W. Humphreys Jr., “Aeroacoustic measurements of a leading edge slat configuration,” International Journal of Aeroacoustics, 1 (3), pp. 241 - 274, 2002.

15. Brooks, T., and W. Humphreys Jr., "A Deconvolution Approach for the Mapping of Acoustic Sources (DAMAS) Determined from Phased Microphone Arrays,” Journal of Sound and Vibration, Volume 294, Issues 4-5, pp. 856-879, July, 2006.

16. Brooks, T., and W. Humphreys Jr., "Three-Dimensional Application of DAMAS Methodology for Aeroacoustic Noise Source Definition,” AIAA paper 2005-2960, presented at the $11^{\text {th }}$ AIAA/CEAS Aeroacoustics Conference, Monterrey, CA, May 23-25, 2005. 